\title{
Analytical Optimization of Piezoelectric Circular Diaphragm Generator
}

\author{
S. Mohammadi ${ }^{1}$ and M. Abdalbeigi ${ }^{2}$ \\ ${ }^{1}$ Mechanical Department, Engineering Faculty, Razi University, Kermanshah 67149-67346, Iran \\ ${ }^{2}$ Mechanical Department, Dareshahr Branch, Islamic Azad University, Dareshahr 6961713751, Iran \\ Correspondence should be addressed to S. Mohammadi; saberm7@yahoo.com
}

Received 14 May 2013; Revised 6 August 2013; Accepted 20 August 2013

Academic Editor: Luciano Mescia

Copyright (c) 2013 S. Mohammadi and M. Abdalbeigi. This is an open access article distributed under the Creative Commons Attribution License, which permits unrestricted use, distribution, and reproduction in any medium, provided the original work is properly cited.

\begin{abstract}
This paper presents an analytical study of the piezoelectric circular diaphragm microgenerator using strain energy method. Piezoelectrics are the intelligent materials that can be used as transducer to convert mechanical energy into electrical energy and vice versa. The aim of this paper is to optimize produced electrical energy from mechanical pressure. Therefore, the circular metal plate equipped with piezoelectric circular patch has been considered with simply and clamped supports. A comprehensive modeling, parametrical study and the effect of the boundary conditions on the performance of the microgenerator have been investigated. The system is under variable pressure from an oscillating pressure source. Results are presented for PZT and PMN-PT piezoelectric materials with steel and aluminum substrates. An optimal value for the radius and thickness of the piezoelectric layer with a special support condition has been obtained.
\end{abstract}

\section{Introduction}

Energy harvesting from environmental sources such as solar, thermal, and mechanical has been the center of interest of some researchers [1-3]. Piezoelectric material can be used as a mechanism to convert mechanical energy into electrical energy and vice versa. If a piezoelectric element is attached to a structure, it is strained as the structure deforms and converts a portion of the mechanical energy into electrical energy that can be harvested through a shunt network. Piezoelectric-based energy harvester can be used as power generator for mobile and low-power consuming devices [417]. Also, these devices present one possible solution to convert ambient vibrations into usable power either to replace the batteries especially in implanted medical devices or to enhance them [18-20]. A hybrid device using magnetic and ultrasonic energies through the magnetostriction and piezoelectric vibrators has been suggested by Suzuki et al. $[21,22]$. Ramsay et al. $[9,23]$ investigated the feasibility of harvesting energy from internal activities in the human body using piezoelectric transducers for microelectromechanical systems applications. This proposed self-powered device provides an in-body power supply.

However, the boundary conditions significantly affect the results [24], several methods have been investigated for vibration energy harvesting using piezoelectric materials without any pointing to these effects. This paper is attempting to address this issue (support conditions) as well as the parametrical study. An analytical approach based on strain energy evaluation is presented in order to model the unimorph piezoelectric circular diaphragm. The source of varying pressure can be the variations of blood pressure, moving vehicles, heartbeat, and other variable pressure sources. The paper has been organized as follows. Section 2 describes the modeling of the system as well as the strain energy calculation. Section 3 depicts the results. Finally, conclusions are provided in Section 4.

\section{Electromechanical Model of Microgenerator}

The energy harvester device is the circular metal plate equipped with piezoelectric circular patch with two boundary 


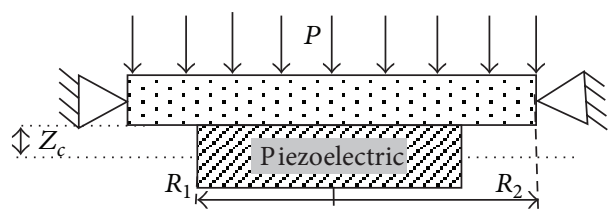

(a)

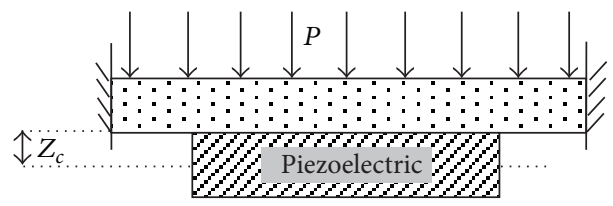

(b)

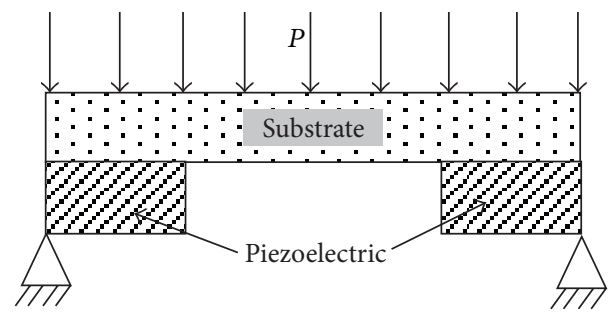

(c)

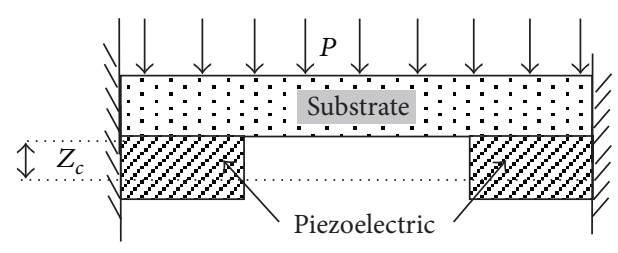

(d)

FIgURE 1: Piezoelectric energy harvester diaphragm.

conditions, simple and clamped supports (Figure 1). The system deflects under applied external pressure and consequently the electrical charge is generated by the piezoelectric diaphragm. Then, this electrical energy can be harvested by a shunt circuit.

The expression of total strain energy in order to calculate the generated energy is required. Therefore, at first, we are going to calculate these equations. The equations of deflection of axially symmetrical plate under uniform pressure $p$ are given by

$$
\begin{gathered}
\frac{1}{r} \frac{d}{d r}\left(r \frac{d}{d r}\left(\frac{1}{r} \frac{d}{d r}\left(r \frac{d w}{d r}\right)\right)\right)=\frac{p}{D}, \\
M_{r}=-D\left(\frac{d^{2} w}{d r^{2}}+\frac{\nu}{r} \frac{d w}{d r}\right), \\
M_{\theta}=-D\left(\frac{1}{r} \frac{d w}{d r}+\nu \frac{d^{2} w}{d r^{2}}\right), \\
Q_{r}=-D \frac{d}{d r}\left(\frac{1}{r} \frac{d}{d r}\left(r \frac{d w}{d r}\right)\right),
\end{gathered}
$$

where $w$ is the plate deflection, $r$ is the radial coordinate, $M_{r}$ and $M_{\theta}$ are the moments in the radial and tangential directions, respectively, $v$ is the poison ratio (assumed to be the same for both layers), $Q_{r}$ is the shear force, and $D$ is the flexural rigidity of the plate. The generated energy by piezoelectric material is calculated using strain energy method in four cases as follows.

2.1. Simply Support. In this case (Figure 1(a)), the plate is divided into two parts: one is the inner section, where $r \leq R_{1}$ and the other is the outer section, where $R_{1}<r \leq R_{2}$. Equations (1)-(3) can be applied for both the inner and outer sections. Inner section is a piezoelectric multilayer plate and outer section is a nonpiezoelectric annular plate. Boundary conditions are as follows:

$$
\begin{gathered}
w_{r=R_{2}}=0, \quad w_{r=R_{1}}^{(i)}=w_{r=R_{1}}^{(o)}, \\
\left.M_{r}\right|_{r=R_{2}}=0,\left.\quad \frac{\partial w^{(i)}}{\partial r}\right|_{r=R_{1}}=\left.\frac{\partial w^{(o)}}{\partial r}\right|_{r=R_{1}}, \\
\left.\frac{\partial w^{(i)}}{\partial r}\right|_{r=0}=0,\left.\quad Q_{r}^{(i)}\right|_{r=R_{1}}=\left.Q_{r}^{(o)}\right|_{r=R_{1}}, \\
\left.M_{r}^{(i)}\right|_{r=R_{1}}=\left.M_{r}^{(o)}\right|_{r=R_{1}} .
\end{gathered}
$$

The subscripts $(i)$ and $(o)$ indicate inner multilayer and outer annular plate, respectively. Deflections of the inner and outer sections are found as

$$
\begin{gathered}
w_{1}=c_{0} r^{4}+c_{1} r^{2}+c_{2} \quad \text { inner section } r \leq R_{1}, \\
w_{2}=c_{6} r^{4}+c_{3} r^{2}+c_{4} \ln r+c_{5} \quad \text { outer section } R_{1}<r<R_{2},
\end{gathered}
$$

where the coefficients of $c_{i}$ have been given in Appendix A.

The flexural rigidities for the inner and outer sections are as follows [25]:

$$
\begin{gathered}
D_{t}=\frac{X Z-Y^{2}}{X}, \\
D=\frac{E I}{1-v^{2}}=\frac{h_{m}^{3}}{12 s_{m}\left(1-v^{2}\right)},
\end{gathered}
$$

where

$$
\begin{aligned}
& X=\sum_{k=1}^{2} \frac{E_{K}}{1-v_{k}^{2}}\left(z_{k}-z_{k-1}\right), \\
& Y=\sum_{k=1}^{2} \frac{E_{K}}{1-v_{k}^{2}}\left(\frac{z_{k}^{2}-z_{k-1}^{2}}{2}\right), \\
& Z=\sum_{k=1}^{2} \frac{E_{K}}{1-v_{k}^{2}}\left(\frac{z_{k}^{3}-z_{k-1}^{3}}{3}\right),
\end{aligned}
$$

where $z_{k}$ is the distance from $k$ th layer to the first layer in any multilayer plate and $E_{K}$ is the young modulus of the $k$ th layer. $I$ is the cross-section moment of inertia, $h_{m}$ is the thickness of substrate layer, and $s_{m}$ is the elastic compliance constant. Strain energy for the piezoelectric layer is

$$
d U_{p}=\frac{1}{2}\left(\varepsilon_{r} \sigma_{r}+\varepsilon_{\theta} \sigma_{\theta}+D_{3} E_{3}\right)
$$


and for the substrate layers

$$
d U_{m}=\frac{1}{2}\left(\varepsilon_{r} \sigma_{r}+\varepsilon_{\theta} \sigma_{\theta}\right) .
$$

The strain equations for piezoelectric material are as follows [26]:

$$
\begin{gathered}
\varepsilon_{r}=s_{11}^{E}\left(\sigma_{r}-\nu \sigma_{\theta}\right)-d_{31} E_{3}, \\
\varepsilon_{\theta}=s_{11}^{E}\left(\sigma_{\theta}-\nu \sigma_{r}\right)-d_{31} E_{3}, \\
D_{3}=-d_{31}\left(\sigma_{r}+\sigma_{\theta}\right)+\varepsilon_{33}^{T} E_{3},
\end{gathered}
$$

where $\sigma, \varepsilon_{33}^{T}, d_{31}, D_{3}$, and $E_{3}$ are the stress, piezoelectric permittivity, piezoelectric constant, charge density, and electric field in 3-direction $(z)$, respectively. The radial direction is taken to be the 1-direction, and tangential direction is the 2direction. The subscripts $r$ and $\theta$ are used instead of 1 and 2, respectively. Substituting of the third equation of (10) into (8) leads to

$$
d U_{p}=\frac{1}{2}\left(\varepsilon_{r} \sigma_{r}+\varepsilon_{\theta} \sigma_{\theta}-d_{31}\left(\sigma_{r}+\sigma_{\theta}\right) E_{3}+\varepsilon_{33}^{T} E_{3}^{2}\right) .
$$

The stress equations for the piezoelectric material can be written as

$$
\begin{gathered}
\sigma_{r}=\frac{1}{s_{11}^{E}\left(1-\nu^{2}\right)}\left(\varepsilon_{r}+\nu \varepsilon_{\theta}+(1+\nu) d_{31} E_{3}\right), \\
\sigma_{\theta}=\frac{1}{s_{11}^{E}\left(1-\nu^{2}\right)}\left(\nu \varepsilon_{r}+\varepsilon_{\theta}+(1+\nu) d_{31} E_{3}\right)
\end{gathered}
$$

and for the nonpiezoelectric material are

$$
\begin{gathered}
\sigma_{r}=\frac{1}{s_{m}\left(1-\nu^{2}\right)}\left(\varepsilon_{r}+\nu \varepsilon_{\theta}\right), \\
\sigma_{\theta}=\frac{1}{s_{m}\left(1-\nu^{2}\right)}\left(\nu \varepsilon_{r}+\varepsilon_{\theta}\right),
\end{gathered}
$$

where $s_{11}^{E}$ is the elastic compliance of piezoelectric material. The equations of curvature and moment (2) can be used for correlating of stress and strain to the applied pressure. The relation of strain and curvature in polar coordinate are as follows:

$$
\begin{aligned}
\varepsilon_{r i}=-\rho_{r i}\left(z-z_{c}\right) & 0 \leq r \leq R_{1}, \\
\varepsilon_{\theta i}=-\rho_{\theta i}\left(z-z_{c}\right) & \\
\varepsilon_{r o}=-\rho_{r o}\left(z-z_{c}\right) & R_{1} \leq r \leq R_{2}, \\
\varepsilon_{\theta o}=-\rho_{\theta o}\left(z-z_{c}\right) &
\end{aligned}
$$

where $\rho_{r}$ and $\rho_{\theta}$ are the radial and tangential curvatures, respectively, and $z_{c}$ is the distance between neutral surface and interface of piezoelectric and substrate layer (Figure 1) that can be written as

$$
z_{c}=\frac{u_{1}}{u_{2}}
$$

where

$$
\begin{gathered}
u_{1}=s_{11}^{E} R_{2} h_{m}^{2}-s_{m} R_{1} h_{p}^{2}, \\
u_{2}=2\left(s_{11}^{E} R_{2} h_{m}+s_{m} R_{1} h_{p}\right),
\end{gathered}
$$

where $h_{p}$ is the thickness of piezoelectric layer. Moment equations for the inner and outer regions can be written as

$$
\begin{gathered}
M_{r i}=\int_{0}^{h_{p}} \sigma_{r p i}\left(z-z_{c}\right) d z+\int_{-h_{m}}^{0} \sigma_{r m i}\left(z-z_{c}\right) d z, \\
M_{\theta i}=\int_{0}^{h_{p}} \sigma_{\theta p i}\left(z-z_{c}\right) d z+\int_{-h_{m}}^{0} \sigma_{\theta m i}\left(z-z_{c}\right) d z, \\
M_{r o}=\int_{-h_{m}}^{0} \sigma_{r m o}\left(z-z_{c}\right) d z, \\
M_{\theta o}=\int_{-h_{m}}^{0} \sigma_{\theta m o}\left(z-z_{c}\right) d z .
\end{gathered}
$$

Substituting of (12) and (13) into the moment (17) leads to

$$
\begin{aligned}
M_{r i}= & \int_{0}^{h_{p}}\left(\frac{\varepsilon_{r i}+v \varepsilon_{\theta i}}{\left(1-v^{2}\right) s_{11}^{E}}+\frac{(1+\nu) d_{31} E_{3}}{\left(1-v^{2}\right) s_{11}^{E}}\right)\left(z-z_{c}\right) d z \\
& +\int_{-h_{m}}^{0}\left(\frac{\varepsilon_{r i}+\nu \varepsilon_{\theta i}}{\left(1-v^{2}\right) s_{m}}\right)\left(z-z_{c}\right) d z, \\
M_{\theta i}= & \int_{0}^{h_{p}}\left(\frac{\nu \varepsilon_{r i}+\varepsilon_{\theta i}}{\left(1-v^{2}\right) s_{11}^{E}}+\frac{(1+\nu) d_{31} E_{3}}{\left(1-v^{2}\right) s_{11}^{E}}\right)\left(z-z_{c}\right) d z \\
& +\int_{-h_{m}}^{0}\left(\frac{\nu \varepsilon_{r i}+\varepsilon_{\theta i}}{\left(1-v^{2}\right) s_{m}}\right)\left(z-z_{c}\right) d z, \\
M_{r o} & =\int_{-h_{m}}^{0}\left(\frac{\varepsilon_{r o}+v \varepsilon_{\theta o}}{\left(1-v^{2}\right) s_{m}}\right)\left(z-z_{c}\right) d z, \\
M_{\theta o} & =\int_{-h_{m}}^{0}\left(\frac{\nu \varepsilon_{r o}+\varepsilon_{\theta o}}{\left(1-v^{2}\right) s_{m}}\right)\left(z-z_{c}\right) d z .
\end{aligned}
$$

By substituting of (14) into (18), the curvature can be found as follows:

$$
\begin{gathered}
\rho_{r i}=\frac{1}{I_{1}\left(1-v^{2}\right)}\left(M_{r i}-v M_{\theta i}\right)+I_{2} E_{3}, \\
\rho_{\theta i}=\frac{1}{I_{1}\left(1-v^{2}\right)}\left(M_{\theta i}-v M_{r i}\right)+I_{2} E_{3}, \\
\rho_{r o}=\frac{1}{I_{3}\left(1-v^{2}\right)}\left(M_{r o}-v M_{\theta o}\right), \\
\rho_{\theta o}=\frac{1}{I_{3}\left(1-v^{2}\right)}\left(M_{\theta o}-v M_{r o}\right),
\end{gathered}
$$

where the coefficients of $I_{i}$ have been given in Appendix A. 
The total strain energy is the combination of the inner and outer strain energies as follows:

$$
\begin{aligned}
U= & \int_{0}^{R_{1}} \int_{0}^{2 \pi} \int_{0}^{h_{p}} d U_{p i} d z r d \theta d r \\
& +\int_{0}^{R_{1}} \int_{0}^{2 \pi} \int_{-h_{m}}^{0} d U_{m i} d z r d \theta d r \\
& +\int_{R_{1}}^{R_{2}} \int_{0}^{2 \pi} \int_{-h_{m}}^{0} d U_{m o} d z r d \theta d r .
\end{aligned}
$$

The first and second terms are for the inner region and the third term is for the outer region. Substituting of (9) and (11) into (20) and using the previous equations of (2), (5), (12)(14), and (19) lead to

$$
U=L_{1} E_{3}^{2}+L_{2} E_{3}+L_{3},
$$

where $L_{1}, L_{2}$, and $L_{3}$ are the constants as given in Appendix A.

It should be mentioned that $M_{r}$ and $M_{\theta}$ in (19) have been replaced by (2) whose deflection has been replaced by (5). Replacing of electric field $E_{3}$ by $V / h_{p}$, where $V$ is the piezoelectric voltage, (21) can be simplified as

$$
U=\alpha V^{2}+\beta V+\gamma
$$

where $=L_{1} / h_{p}^{2}, \beta=L_{2} / h_{p}$, and $\gamma=L_{3}$. The term $\alpha V^{2}$ represents the electrical energy stored on the capacitance of the piezoelectric material due to an externally applied voltage, which does not exist in this case. $\gamma$ represents the mechanical energy, and $\beta V$ is the converted energy from mechanical deformation to electrical energy, which can be harvested. The aim is to maximize this term. The total electrical charge $Q_{t}$ can be obtained by differentiating of (22) with respect to voltage $V$ :

$$
Q_{t}=\frac{\partial U}{\partial V}=2 \alpha V+\beta
$$

Thus, $\beta$ is the charge generated $\left(Q_{\text {gen }}\right)$ by the applied pressure. From the terms $2 \alpha V$ and $Q=C_{\text {free }} V$ for the capacitance, it can be concluded that

$$
C_{\text {free }}=2 \alpha .
$$

Then

$$
V_{\text {gen }}=\frac{Q_{\text {gen }}}{C_{\text {free }}},
$$

and then

$$
V_{\text {gen }}=\frac{\beta}{2 \alpha}
$$

where $C_{\text {free }}$ is the open-circuit piezocapacitance $V_{\text {gen }}$ is the voltage generated by the applied pressure $p$. Therefore, the electrical energy that is generated by the applied external pressure $p$ is

$$
U_{\text {gen }}=\frac{Q_{\text {gen }} V_{\text {gen }}}{2} \text {, }
$$

and then

$$
U_{\text {gen }}=\frac{\beta^{2}}{4 \alpha}
$$

2.2. Clamped Support. In this case, a similar method as in Section 2.1 is applied only with different boundary conditions (Figure 1(b)). The support in this case is clamped, and the boundary conditions are as follows:

$$
\begin{gathered}
w_{r=R_{2}}=0,\left.\quad \frac{\partial w^{(o)}}{\partial r}\right|_{r=R_{2}}=0, \quad w_{r=R_{1}}^{(i)}=w_{r=R_{1}}^{(o)}, \\
\left.\frac{\partial w^{(i)}}{\partial r}\right|_{r=R_{1}}=\left.\frac{\partial w^{(o)}}{\partial r}\right|_{r=R_{1}},\left.\quad \frac{\partial w^{(i)}}{\partial r}\right|_{r=0}=0, \\
\left.Q_{r}^{(i)}\right|_{r=R_{1}}=\left.Q_{r}^{(o)}\right|_{r=R_{1}},\left.\quad M_{r}^{(i)}\right|_{r=R_{1}}=\left.M_{r}^{(o)}\right|_{r=R_{1}} .
\end{gathered}
$$

Deflections of the inner and outer sections are found as follows:

$$
\begin{gathered}
w_{1}=c_{0} r^{4}+c_{1} r^{2}+c_{2} \quad \text { inner section } r \leq R_{1} \\
w_{2}=c_{6} r^{4}+c_{3} r^{2}+c_{4} \ln r+c_{5} \quad \text { outer section } R_{1}<r<R_{2}
\end{gathered}
$$

where $c_{i}$ are the coefficients similar to those in (5) with $A_{1}$ and $A_{2}$ as the following

$$
\begin{aligned}
& A_{1}=\frac{b_{19} b_{4}-b_{18} b_{16}}{b_{20} b_{16}-b_{19} b_{17}} \\
& A_{2}=\frac{-\left(b_{18}+b_{20} A_{1}\right)}{b_{19}}
\end{aligned}
$$

where the coefficients of $b_{i}$ have been given in Appendix A.

The equation of energy in this case is similar to (21) with the same constants of $L_{1}, L_{2}$, and $L_{3}$. Thus, the remaining calculations are similar to those in Section 2.1 from (22) to (28).

2.3. System with Annular Piezoelectric Plate and Simple Support. In this case (Figure 1(c)), the plate is divided into two parts: one is the inner section, where $r \leq R_{1}$ and the other is the outer section, where $R_{1}<r \leq R_{2}$. Equations (1)-(3) can be applied for both the inner and outer sections. Outer section is a piezoelectric multilayer annular plate and inner section is a nonpiezoelectric plate. Boundary conditions are similar to (4). Deflections of the inner and outer sections can be found as

$$
\begin{gathered}
w_{1}=c_{0} r^{4}+c_{1} r^{2}+c_{2} \quad \text { inner section } r \leq R_{1} \\
w_{2}=c_{6} r^{4}+c_{3} r^{2}+c_{4} \ln r+c_{5} \quad \text { outer section } R_{1}<r<R_{2}
\end{gathered}
$$


where the coefficients of $c_{i}$ have been given in Appendix B. The total energy is the combination of the inner and outer strain energies as follows:

$$
\begin{aligned}
U= & \int_{R_{1}}^{R_{2}} \int_{0}^{2 \pi} \int_{0}^{h_{p}} d U_{p o} d z r d \theta d r \\
& +\int_{R_{1}}^{R_{2}} \int_{0}^{2 \pi} \int_{-h_{m}}^{0} d U_{m o} d z r d \theta d r \\
& +\int_{0}^{R_{1}} \int_{0}^{2 \pi} \int_{-h_{m}}^{0} d U_{m i} d z r d \theta d r .
\end{aligned}
$$

Substituting of (9) and (11) into this equation and using the previous equations of (2), (12)-(14), (19), and (32) lead to

$$
U=L_{1} E_{3}^{2}+L_{2} E_{3}+L_{3},
$$

where $L_{1}, L_{2}$, and $L_{3}$ are the constants as given in Appendix B. The remaining equations are similar to those in Section 2.1 from (22) to (28).

2.4. System with Annular Piezoelectric Plate and Clamped Support. In this case, a similar method as in Section 2.3 is applied only with different boundary conditions. (Figure 1(d)). The support in this case is clamped and the boundary conditions are similar to those in Section 2.2. Thus, the deflections of the inner and outer sections are similar to (30) with their constants of $c_{i}$ similar to those in (32) with $A_{1}$ and $A_{2}$ as follows:

$$
\begin{aligned}
& A_{1}=\frac{b_{19} b_{4}-b_{18} b_{16}}{b_{20} b_{16}-b_{19} b_{17}}, \\
& A_{2}=\frac{-\left(b_{18}+b_{20} A_{1}\right)}{b_{19}},
\end{aligned}
$$

where the coefficients of $b_{i}$ have been given in Appendix B. Equation of energy in this case is similar to (34) with the same constants of $L_{1}, L_{2}$, and $L_{3}$. The remaining equations are similar to those in Section 2.1 from (22) to (28).

\section{Results and Discussions}

In this paper, the strain energy solution is used in order to paramedical studies, effects of support conditions and substrate material on the generated power by the circular piezoelectric diaphragm. The substrate materials are the circular metal plate of aluminum and steel that are equipped with piezoelectric material. The excitation signal is a uniform pressure of $40 \mathrm{mmHg}(5330 \mathrm{~Pa})$ with $1 \mathrm{~Hz}$ frequency, which could arise from a blood pressure source. Table 1 summarizes materials' properties that are used in this section.

Figures 2 and 3 show the variations of generated energy versus the radius ratio $\left(R_{1} / R_{2}\right)$ for each support condition. It

\begin{tabular}{|c|c|c|c|c|}
\hline $\begin{array}{l}\varepsilon_{33}^{T} \\
\left(\times 10^{-12}\right)\end{array}$ & $\begin{array}{c}S_{11}^{E}, S_{m} \\
\left(\times 10^{-12} \mathrm{~m}^{2} \mathrm{~N}^{-1}\right)\end{array}$ & $v$ & $\begin{array}{c}d_{31} \\
\left(\times 10^{-12} \mathrm{mV}\right)\end{array}$ & Materials \\
\hline 30090 & 16.5 & 0.3 & -274 & PZT-5H \\
\hline 48675 & 1.25 & 0.3 & -1063 & PMN-\%33PT \\
\hline- & 0.14286 & - & - & Aluminum \\
\hline- & 5 & - & - & Steel \\
\hline
\end{tabular}
can be seen that the generated energy has an optimum value related to piezoelectric plate radius $R_{1} / R_{2}$. Also, the effect of boundary conditions on the generated energy is evident. It can be concluded that harvested energy in the case of simple
TABLE 1: Piezoelectric and substrate materials properties.

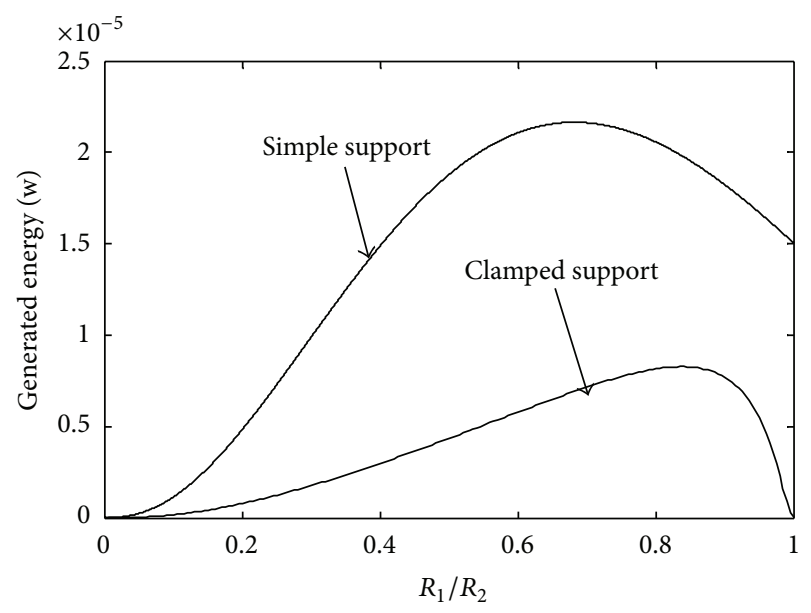

FIGURE 2: Generated energy for the simple and clamped supports according to the variations of piezoelectric plate radius $\left(R_{2}=\right.$ $20 \mathrm{~mm}$ ) (Sections 2.1 and 2.2).

support is more than clamped support for the same conditions (same PZT piezoelectric thickness $350 \mu \mathrm{m}$, applied pressure $40 \mathrm{mmHg}$, and aluminum substrate material with $175 \mu \mathrm{m}$ thickness). The piezoelectric material consumption in the case of simple support is less than clamped support because its optimum value of $R_{1} / R_{2}$ is smaller than clamped support (Figure 2). This optimum value of $R_{1} / R_{2}$ in Figure 3 for simple support is greater than clamped support. Thus, in this case, the piezoelectric material consumption is also less for simple support condition. In each curve, harvested energy with increase of piezoelectric material increases, but in contrast, the bending resistance increases as well, consequently resulting in less deflection and then less generated energy. By this way, each curve has an optimum value (maximum value).

Figure 4 shows the effect of piezoelectric thickness on the generated energy in the case of simple support condition (Section 2.1). It is observed that generated power has an optimum value related to piezoelectric thickness variations. It increases with increase of piezoelectric thickness, but in contrast the bending resistance increases as well, consequently resulting in less deflection and then less harvested energy. It should be mentioned that piezoelectric power is proportional to deflection. Thus, generated power should be in good agreement with the piezoelectric thickness and radius. The results have been calculated with the outer radius of $20 \mathrm{~mm}$, AL substrate plate thickness of $175 \mu \mathrm{m}$, PZT material and 


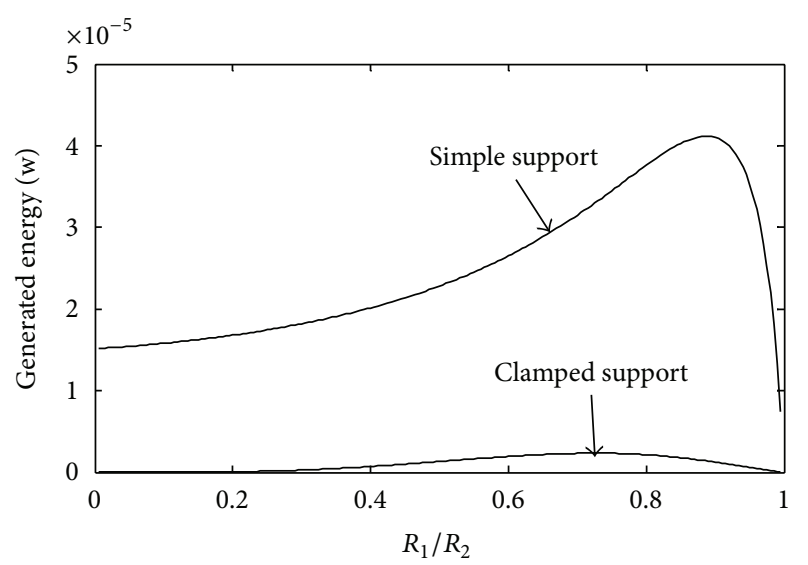

FIGURE 3: Generated energy for the simple and clamped supports according to the variations of piezoelectric plate radius $\left(R_{2}=\right.$ $20 \mathrm{~mm}$ ) (Sections 2.3 and 2.4).

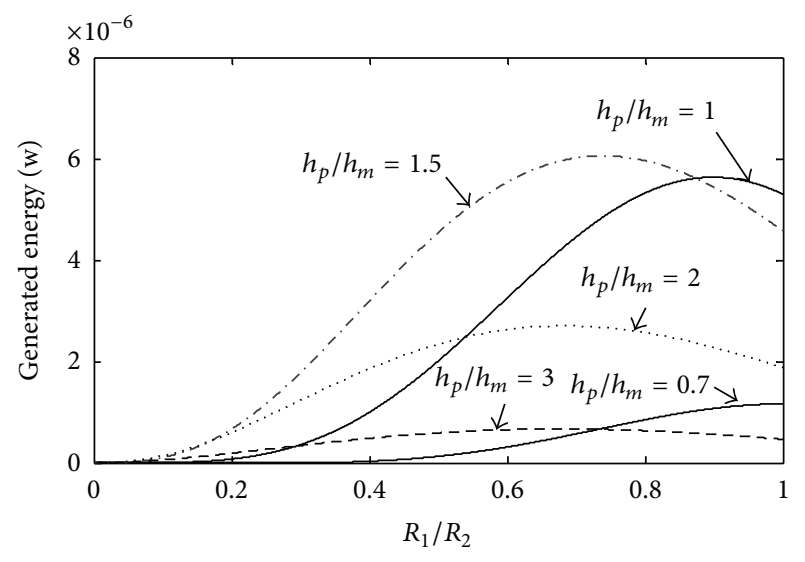

FIGURE 4: Effect of piezoelectric thickness on generated energy in the case of simple support (Section 2.1).

applied pressure of $5330 \mathrm{pa}$. The harvested energy for $h_{p} / h_{m}=$ 1.5 and $R_{1} / R_{2}=0.72$ is maximum and equal to $6 \mu \mathrm{W}$.

Figure 5 shows the generated energy that is provided by different piezoelectric materials in the case of simple support condition (Section 2.1). To investigate this effect, two materials of PMN-PT and PZT have been considered. It is observed that generated power is sensitive to the type of piezoelectric material. In these results, the piezoelectric thickness is $350 \mu \mathrm{m}$ with aluminum substrate material of $175 \mu \mathrm{m}$ thickness.

Also, the material substrate affects the results. This has been shown in Figure 6 generated energy for the aluminum substrate is more than steel substrate. Since the aluminum stiffness is less than steel stiffness, its deflection is more and consequently it provided more generated energy.

\section{Conclusions}

An analytical model using strain energy method for piezoelectric circular diaphragm generator has been presented. A parametrical study to investigate the effects of various parameters on the generated power has been performed.

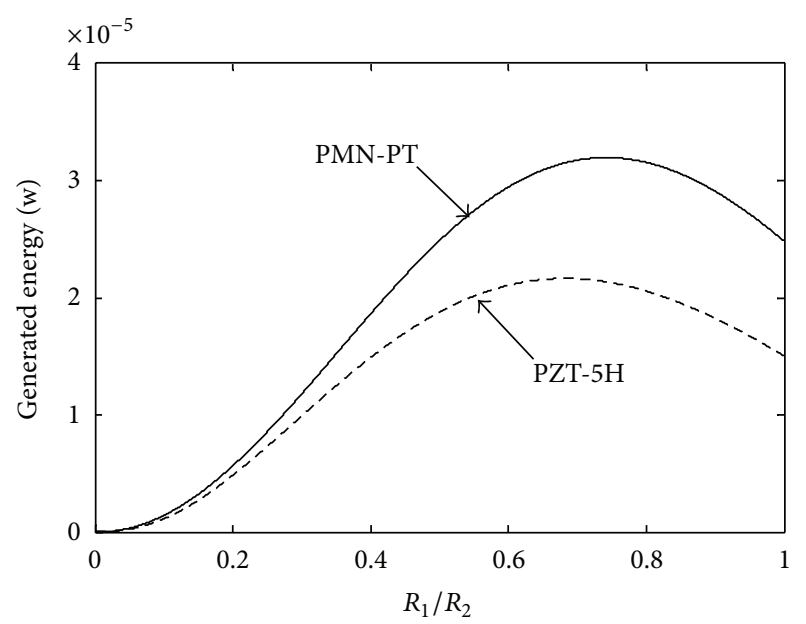

FIGURE 5: Effect of piezoelectric material on generated energy in the case of simple support $\left(R_{2}=20 \mathrm{~mm}\right)$ (Section 2.1).

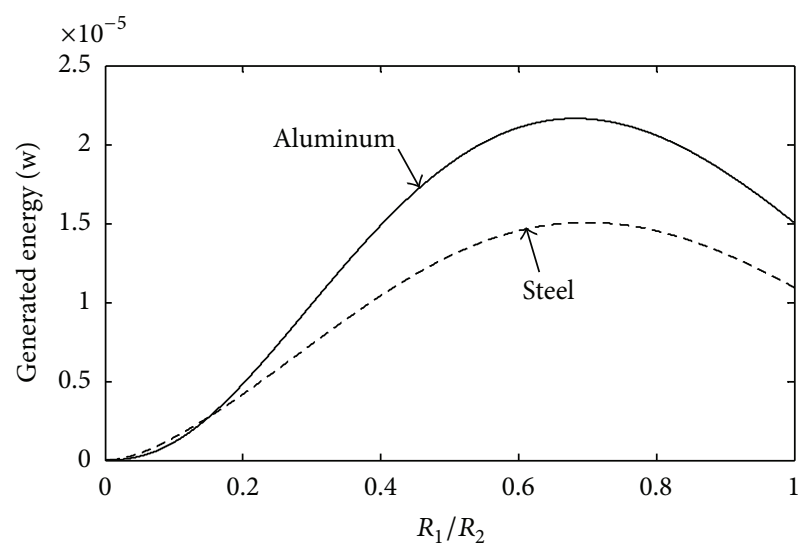

FIGURE 6: Effect of substrate material on generated energy with simple support conditions (Section 2.1).

With the proposed model, the generated power has been compared in four cases of simple and clamped supports conditions under uniformly distributed pressure. It was shown that the boundary conditions, material, and radius and thickness of piezoelectric layer and substrate material affect the results. Therefore, generated power should be in good agreement with the piezoelectric thickness and radius with a special support condition.

\section{Appendices}

\section{A.}

Coefficients of (5). Consider

$$
\begin{gathered}
c_{0}=\frac{p}{64 D_{t}}, \quad c_{1}=F_{1}, \\
c_{2}=A_{3}-b_{3} A_{1}+b_{2} A_{2}-b_{2} F_{1}-b_{1}, \\
c_{3}=A_{2}, \quad c_{4}=A_{1}, \\
c_{5}=A_{3}, \quad c_{6}=\frac{p}{64 D},
\end{gathered}
$$


where

$$
\begin{gathered}
A_{1}=\frac{b_{4} b_{13}-b_{12} b_{16}}{b_{14} b_{16}-b_{13} b_{17}}, \quad A_{2}=\frac{-\left(b_{12}+b_{14} A_{1}\right)}{b_{13}}, \\
A_{3}=-\left(b_{7}+b_{8} A_{2}+b_{9} A_{1}\right), \quad F_{1}=\frac{-b_{15}}{b_{10}} A_{2}-\frac{b_{11}}{b_{10}} A_{1}, \\
b_{1}=\frac{p R_{1}^{4}}{64}\left(\frac{1}{D_{t}}-\frac{1}{D}\right), \quad b_{2}=R_{1}^{2}, \\
b_{3}=-\ln R_{1}, \quad b_{4}=\frac{p R_{1}^{3}}{16}\left(\frac{1}{D_{t}}-\frac{1}{D}\right), \quad b_{5}=2 R_{1}, \\
b_{6}=\frac{-1}{R_{1}}, \quad b_{7}=\frac{p R_{2}^{4}}{64 D}, \quad b_{8}=R_{2}^{2}, \\
b_{9}=\ln R_{2}, \quad b_{10}=2(1+\nu) D_{t}, \quad b_{11}=\frac{D}{R_{1}^{2}}(1-\nu), \\
b_{12}=\frac{-p}{16}(3+\nu) R_{2}^{2}, \quad b_{13}=-2 D(1+\nu), \\
b_{15}=b_{13}, \quad b_{14}=\frac{D}{R_{2}^{2}}(1-\nu), \\
b_{16}=\frac{-b_{5}\left(b_{15}+b_{10}\right)}{b_{10}}, \quad b_{18}=\frac{p}{16 D} R_{2}^{3}, \\
b_{19}=2 R_{2}, \quad b_{20}=\frac{1}{R_{2}} .
\end{gathered}
$$

Coefficients of (19). Consider

$$
\begin{gathered}
I_{1}=\left(\left(s_{m} h_{p}^{3}+s_{11}^{E} h_{m}^{3}\right) u_{2}^{2}+3\left(s_{11}^{E} h_{m}^{2}-s_{m} h_{p}^{2}\right) u_{1} u_{2}\right. \\
\left.+3\left(s_{11}^{E} h_{m}-s_{m} h_{p}\right) u_{1}^{2}\right) \times\left(3\left(1-v^{2}\right) s_{11}^{E} s_{m} u_{2}^{2}\right)^{-1}, \\
I_{2}=\frac{d_{31}\left(h_{p}^{2} u_{2}-2 h_{p} u_{1}\right)}{2 u_{2}(1-v) s_{11}^{E}}, \\
I_{3}=\frac{h_{m}^{3} u_{2}^{2}+3 h_{m}^{2} u_{1} u_{2}+3 h_{m} u_{1}^{2}}{3 u_{2}^{2}\left(1-v^{2}\right) s_{m}}, \quad I_{4}=\frac{-I_{2}}{I_{1}(1+\nu)} .
\end{gathered}
$$

Coefficients of (21). Consider

$$
L_{1}=G_{1}+G_{4}, \quad L_{2}=G_{2}+G_{5}, \quad L_{3}=G_{3}+G_{6}+G_{7},
$$

where

$$
\begin{aligned}
G_{1}= & \pi h_{p} R_{1}^{2}\left(\frac{\varepsilon_{33}^{T}}{2}-e_{4} d_{31}\right) \\
& +\pi H_{1}\left(k_{9}-e_{3} d_{31}\right) R_{1}^{2}+\pi H_{2} k_{6} R_{1}^{2}, \\
G_{2}= & \pi H_{1} R_{1}^{4}\left(\frac{k_{8}+k_{13}-\left(e_{2}+e_{5}\right) d_{31}}{4}\right) \\
& +\pi H_{1}\left(k_{7}-e_{1} d_{31}\right) \\
& +\pi H_{2} R_{1}^{4}\left(\frac{k_{4}+k_{11}}{4}\right)+\pi H_{2} k_{3} R_{1}^{2},
\end{aligned}
$$

$$
\begin{gathered}
G_{3}=\pi H_{2} k_{1} R_{1}^{2}+\pi H_{2} R_{1}^{4}\left(\frac{k_{2}+k_{10}}{4}\right)+\pi H_{2} R_{1}^{6}\left(\frac{k_{5}+k_{12}}{6}\right), \\
G_{4}=\pi H_{3} q_{6} R_{1}^{2}, \\
G_{5}=\pi H_{3} q_{3} R_{1}^{2}+\pi H_{3} R_{1}^{4}\left(\frac{q_{4}+q_{8}}{4}\right), \\
G_{6}=\pi H_{3} q_{1} R_{1}^{2}+\pi H_{3} R_{1}^{4}\left(\frac{q_{2}+q_{7}}{4}\right) \\
+\pi H_{3} R_{1}^{6}\left(\frac{q_{5}+q_{9}}{6}\right), \\
G_{7}=2 \pi H_{3}\left[\left(\frac{x_{1}+x_{6}}{2}\right) V_{5}+\left(\frac{x_{2}+x_{7}}{2}\right) V_{3}\right. \\
+\left(\frac{x_{3}+x_{8}}{2}\right) V_{4}+\left(\frac{x_{4}+x_{9}}{2}\right) V_{1} \\
\left.+\left(\frac{x_{5}+x_{10}}{2}\right) V_{2}\right],
\end{gathered}
$$

where

$$
\begin{gathered}
x_{1}=-\left(B_{1} g_{1}+B_{2} g_{3}+B_{3} g_{2}\right), \quad x_{2}=-\left(B_{1} g_{2}+B_{2} g_{1}\right), \\
x_{3}=-\left(B_{1} g_{3}+B_{3} g_{1}\right), \quad x_{4}=-\left(B_{2} g_{2}\right), \\
x_{5}=-\left(B_{3} g_{3}\right), \quad x_{6}=-\left(B_{1} g_{1}+B_{4} g_{5}+B_{5} g_{4}\right), \\
x_{7}=-\left(B_{1} g_{4}+B_{4} g_{1}\right), \quad x_{8}=-\left(B_{1} g_{5}+B_{5} g_{1}\right), \\
x_{9}=-\left(B_{4} g_{4}\right), \quad x_{10}=-\left(B_{5} g_{5}\right), \\
H_{1}=h_{p}\left(\frac{h_{p}}{2}-z_{c}\right), \quad H_{2}=\frac{\left(h_{p}-z_{c}\right)^{3}-z_{c}^{3}}{3}, \\
H_{3}=\frac{\left(h_{m}+z_{c}\right)^{3}-z_{c}^{3}}{3}, \\
q_{1}=-\left(B_{6} f_{1}\right), \quad q_{2}=-\left(B_{7} f_{1}+B_{6} f_{2}\right), \\
q_{3}=-\left(I_{4} f_{1}+B_{6} f_{3}\right), \quad q_{4}=-\left(B_{7} f_{3}+I_{4} f_{2}\right), \\
q_{5}=-B_{7} f_{2}, \quad q_{6}=-I_{4} f_{3}, \\
q_{7}=-\left(B_{8} f_{1}+B_{6} f_{4}\right), \quad q_{8}=-\left(I_{4} f_{4}+B_{8} f_{3}\right), \\
q_{9}=-B_{8} f_{4}, \quad k_{1}=-\left(B_{6} e_{1}\right), \\
k_{2}=-\left(B_{7} e_{1}+B_{6} e_{2}\right), \quad k_{3}=-\left(I_{4} e_{1}+B_{6} e_{3}\right), \\
k_{4}=-\left(B_{7} e_{3}+I_{4} e_{2}\right), \quad k_{5}=-B_{7} e_{2} \\
k_{6}=-I_{4} e_{3}, \quad k_{7}=-\left(B_{6} e_{4}\right), \\
k_{8}=-\left(B_{7} e_{4}\right), \quad k_{9}=-\left(I_{4} e_{4}\right), \\
\left.k_{1}=-B B_{1}(1+\nu), \quad B_{8} e_{1}+B_{6} e_{5}\right), \quad k_{11}=-\left(I_{4} e_{5}+B_{8} e_{3}\right), \\
k_{12}=-\left(B_{8} e_{5}\right), \quad k_{13}=-\left(B_{8} e_{4}\right),
\end{gathered}
$$




$$
\begin{aligned}
& g_{3}=-B\left(B_{3}+v B_{5}\right), \quad g_{4}=-B\left(B_{4}+v B_{2}\right) \\
& g_{5}=-B\left(B_{5}+v B_{3}\right), \quad f_{1}=-B B_{6}(1+\nu), \\
& f_{2}=-B\left(B_{7}+\nu B_{8}\right), \quad f_{3}=-B I_{4}(1+\nu), \\
& f_{4}=-B\left(B_{8}+v B_{7}\right), \quad e_{1}=-A B_{6}(1+v), \\
& e_{2}=-A\left(B_{7}+v B_{8}\right), \quad e_{3}=-A I_{4}(1+v) \text {, } \\
& e_{4}=A d_{31}(1+v), \quad e_{5}=-A\left(B_{8}+v B_{7}\right), \\
& A=\frac{1}{s_{11}^{E}\left(1-v^{2}\right)}, \quad B=\frac{1}{s_{m}\left(1-v^{2}\right)}, \\
& B_{1}=I_{6} a_{4}(1-v), \quad B_{2}=I_{6}\left(a_{5}-v a_{6}\right), \\
& B_{3}=I_{6}\left(a_{7}-v a_{8}\right), \quad B_{4}=I_{6}\left(a_{6}-v a_{5}\right), \\
& B_{5}=I_{6}\left(a_{8}-v a_{7}\right), \quad B_{6}=a_{1} I_{5}(1-v), \\
& B_{7}=I_{5}\left(a_{2}-v a_{3}\right), \quad B_{8}=I_{5}\left(a_{3}-v a_{2}\right), \\
& a_{1}=-2 c_{1} D_{t}(1+\nu), \quad a_{2}=-4 c_{0} D_{t}(3+\nu) \text {, } \\
& a_{3}=-4 c_{0} D_{t}(1+3 v), \quad a_{4}=-2 c_{3} D(1+\nu) \text {, } \\
& a_{5}=-4 c_{6} D(3+v), \quad a_{6}=-4 c_{6} D(1+3 v) \text {, } \\
& a_{7}=c_{4} D(1-v), \quad a_{8}=c_{4} D(\nu-1), \\
& V_{1}=\frac{1}{6}\left(R_{2}^{6}-R_{1}^{6}\right), \quad V_{2}=\frac{1}{2 R_{1}^{2}}-\frac{1}{2 R_{2}^{2}}, \\
& V_{3}=\frac{1}{4}\left(R_{2}^{4}-R_{1}^{4}\right), \quad V_{4}=\ln \frac{R_{2}}{R_{1}}, \\
& V_{5}=\frac{1}{2}\left(R_{2}^{2}-R_{1}^{2}\right), \quad I_{5}=\frac{1}{I_{1}\left(1-v^{2}\right)}, \\
& I_{6}=\frac{-B D}{I_{3}} .
\end{aligned}
$$

\section{B.}

Coefficients of (32). Consider

$$
\begin{gathered}
c_{0}=\frac{p}{64 D}, \quad c_{1}=F_{1}, \\
c_{2}=A_{3}-b_{3} A_{1}+b_{2} A_{2}-b_{2} F_{1}-b_{1}, \\
c_{3}=A_{2}, \quad c_{4}=A_{1}, \quad c_{5}=A_{3}, \quad c_{6}=\frac{p}{64 D_{t}},
\end{gathered}
$$

where

$$
\begin{gathered}
A_{1}=\frac{b_{4} b_{13}-b_{12} b_{16}}{b_{14} b_{16}-b_{13} b_{17}}, \quad A_{2}=\frac{-\left(b_{12}+b_{14} A_{1}\right)}{b_{13}}, \\
F_{1}=\frac{-b_{15}}{b_{10}} A_{2}-\frac{b_{11}}{b_{10}} A_{1},
\end{gathered}
$$

$$
\begin{gathered}
b_{1}=\frac{-p R_{1}^{4}}{64}\left(\frac{1}{D_{t}}-\frac{1}{D}\right), \quad b_{2}=R_{1}^{2}, \quad b_{3}=-\ln R_{1}, \\
b_{4}=\frac{-p R_{1}^{3}}{16}\left(\frac{1}{D_{t}}-\frac{1}{D}\right), \quad b_{5}=2 R_{1}, \\
b_{6}=\frac{-1}{R_{1}}, \quad b_{7}=\frac{p R_{2}^{4}}{64 D_{t}}, \quad b_{8}=R_{2}^{2}, \\
b_{9}=\ln R_{2}, \quad b_{10}=2(1+v) D, \\
b_{11}=\frac{D_{t}}{R_{1}^{2}}(1-v), \quad b_{12}=\frac{-p}{16}(3+v) R_{2}^{2}, \\
b_{13}=-2 D_{t}(1+\nu), \quad b_{14}=\frac{D_{t}}{R_{2}^{2}}(1-v), \\
b_{15}=b_{13}, \quad b_{16}=\frac{-b_{5}\left(b_{15}+b_{10}\right)}{b_{10}}, \\
b_{17}=\frac{b_{6} b_{10}-b_{5} b_{11}}{b_{10}}, \quad \begin{array}{c}
b_{18}=\frac{p}{16 D} R_{2}^{3}, \\
b_{19}=2 R_{2}, \quad b_{20}=\frac{1}{R_{2}} .
\end{array}
\end{gathered}
$$

Coefficients of (34). Consider

$$
L_{1}=G_{1}+G_{4}, \quad L_{2}=G_{2}+G_{5}, \quad L_{3}=G_{3}+G_{6}+G_{7},
$$

where

$$
\begin{aligned}
G_{1}= & 2 \pi h_{p} V_{5}\left(\frac{\varepsilon_{33}^{T}}{2}-e_{5} d_{31}\right)+2 \pi H_{1}\left(k_{13}-e_{4} d_{31}\right) V_{5} \\
& +2 \pi H_{2} k_{9} V_{5}, \\
G_{2}= & 2 \pi H_{2} k_{4} V_{5}+\pi H_{2}\left(k_{5}+k_{17}\right) V_{3}+\pi H_{2}\left(k_{6}+k_{18}\right) V_{4} \\
& +2 \pi H_{1}\left(k_{10}-e_{1} d_{31}\right) V_{5} \\
& +\pi H_{1}\left(k_{11}+k_{21}-\left(e_{2}+e_{6}\right) d_{31}\right) V_{3} \\
& +\pi H_{1}\left(k_{12}+k_{22}-\left(e_{3}+e_{7}\right) d_{31}\right) V_{4}, \\
G_{3}= & H_{2}\left[\left(k_{1}+k_{14}\right) V_{5}+\left(k_{2}+k_{15}\right) V_{3}\right. \\
& \left.+\left(k_{3}+k_{16}\right) V_{4}+\left(k_{7}+k_{19}\right) V_{1}+\left(k_{8}+k_{20}\right) V_{2}\right], \\
G_{5}= & 2 \pi H_{3} q_{4} V_{5}+\pi H_{3}\left(q_{5}+q_{13}\right) V_{3}+\pi H_{3}\left(q_{6}+q_{14}\right) V_{4}, \\
G_{6}= & \pi H_{3}\left[\left(q_{1}+q_{10}\right) V_{5}+\left(q_{2}+q_{11}\right) V_{3}+\left(q_{3}+q_{12}\right) V_{4}\right. \\
& \left.+\left(q_{7}+q_{15}\right) V_{1}+\left(q_{8}+q_{16}\right) V_{2}\right], \\
G_{7}= & \pi H_{3}\left[x_{1} R_{1}^{2}+\left(x_{2}+x_{4}\right) \frac{R_{1}^{4}}{4}+\left(x_{3}+x_{5}\right) \frac{R_{1}^{6}}{4}\right],
\end{aligned}
$$


where

$$
\begin{aligned}
& x_{1}=-\left(B_{6} g_{1}\right), \quad x_{2}=-\left(B_{7} g_{1}+B_{6} g_{2}\right), \\
& x_{3}=-\left(B_{7} g_{2}\right), \quad x_{4}=-\left(B_{8} g_{1}+B_{6} g_{3}\right) \text {, } \\
& x_{5}=-\left(B_{8} g_{3}\right), \quad q_{1}=-\left(B_{1} f_{1}+B_{2} f_{3}+B_{3} f_{2}\right) \text {, } \\
& q_{2}=-\left(B_{1} f_{2}+B_{2} f_{1}\right), \quad q_{3}=-\left(B_{1} f_{3}+B_{3} f_{1}\right), \\
& q_{4}=-\left(B_{1} f_{4}+I_{4} f_{1}\right), \quad q_{5}=-\left(B_{2} f_{4}+I_{4} f_{2}\right), \\
& q_{6}=-\left(B_{3} f_{4}+I_{4} f_{3}\right), \quad q_{7}=-\left(B_{2} f_{2}\right), \\
& q_{8}=-\left(B_{3} f_{3}\right), \quad q_{9}=-I_{4} f_{4}, \\
& q_{10}=-\left(B_{1} f_{1}+B_{4} f_{6}+B_{5} f_{5}\right), \quad q_{11}=-\left(B_{1} f_{5}+B_{4} f_{1}\right) \text {, } \\
& q_{12}=-\left(B_{1} f_{6}+B_{5} f_{1}\right), \quad q_{13}=-\left(B_{4} f_{4}+I_{4} f_{5}\right) \text {, } \\
& q_{14}=-\left(B_{5} f_{4}+I_{4} f_{6}\right), \quad q_{15}=-\left(B_{4} f_{5}\right), \\
& q_{16}=-\left(B_{5} f_{6}\right), \quad k_{1}=-\left(B_{1} e_{1}+B_{2} e_{3}+B_{3} e_{2}\right), \\
& k_{2}=-\left(B_{1} e_{2}+B_{2} e_{1}\right), \quad k_{3}=-\left(B_{1} e_{3}+B_{3} e_{1}\right), \\
& k_{4}=-\left(B_{1} e_{4}+I_{4} e_{1}\right), \quad k_{5}=-\left(B_{2} e_{4}+I_{4} e_{2}\right), \\
& k_{6}=-\left(B_{3} e_{4}+I_{4} e_{3}\right), \quad k_{7}=-\left(B_{2} e_{2}\right), \\
& k_{8}=\left(-B_{3} e_{3}\right), \quad k_{9}=-\left(I_{4} e_{4}\right), \\
& k_{10}=-\left(B_{1} e_{5}\right), \quad k_{11}=-\left(B_{2} e_{5}\right) \\
& k_{12}=-\left(B_{3} e_{5}\right), \quad k_{13}=-\left(I_{4} e_{5}\right), \\
& k_{14}=-\left(B_{1} e_{1}+B_{4} e_{7}+B_{5} e_{4}\right), \quad k_{15}=-\left(B_{1} e_{6}+B_{4} e_{1}\right) \text {, } \\
& k_{16}=-\left(B_{1} e_{7}+B_{5} e_{1}\right), \quad k_{17}=-\left(B_{4} e_{4}+I_{4} e_{6}\right), \\
& k_{18}=-\left(B_{5} e_{4}+I_{4} e_{7}\right), \quad k_{19}=-\left(B_{4} e_{6}\right), \\
& k_{20}=-\left(B_{5} e_{5}\right), \quad k_{21}=-\left(B_{4} e_{5}\right), \quad k_{22}=k_{20}, \\
& g_{1}=-B B_{6}(1+\nu), \quad g_{2}=-B\left(B_{7}+v B_{8}\right), \\
& g_{3}=-B\left(B_{8}+v B_{7}\right), \\
& f_{1}=-B B_{1}(1+\nu), \quad f_{2}=-B\left(B_{2}+\nu B_{4}\right), \\
& f_{3}=-B\left(B_{3}+\nu B_{5}\right), \quad f_{4}=-B I_{4}(1+\nu), \\
& f_{5}=-B\left(B_{4}+v B_{2}\right), \quad f_{6}=-B\left(B_{5}+v B_{3}\right), \\
& e_{1}=-A B_{1}(1+\nu), \quad e_{2}=-A\left(B_{2}+\nu B_{4}\right), \\
& e_{3}=-A\left(B_{3}+\nu B_{5}\right), \quad e_{4}=-A I_{4}(1+\nu), \\
& e_{5}=A d_{31}(1+v), \quad e_{6}=-A\left(B_{4}+v B_{2}\right), \\
& e_{7}=-A\left(B_{5}+v B_{3}\right), \quad A=\frac{1}{s_{11}^{E}\left(1-v^{2}\right)}, \\
& B=\frac{1}{s_{m}\left(1-v^{2}\right)},
\end{aligned}
$$

$$
\begin{array}{cl}
B_{1}=I_{5} a_{4}(1-v), & B_{2}=I_{5}\left(a_{5}-v a_{6}\right), \\
B_{3}=I_{5}\left(a_{7}-v a_{8}\right), & B_{4}=I_{5}\left(a_{6}-v a_{5}\right), \\
B_{5}=I_{5}\left(a_{8}-v a_{7}\right), & B_{6}=a_{1} I_{6}(1-v), \\
B_{7}=I_{6}\left(a_{2}-v a_{3}\right), & B_{8}=I_{6}\left(a_{3}-v a_{2}\right), \\
a_{1}=-2 c_{1} D(1+v), & a_{2}=-4 c_{0} D(3+v), \\
a_{3}=-4 c_{0} D(1+3 v), & a_{4}=-2 c_{3} D_{t}(1+v), \\
a_{5}=-4 c_{6} D_{t}(3+v), & a_{6}=-4 c_{6} D_{t}(1+3 v), \\
a_{7}=c_{4} D_{t}(1-v), & a_{8}=c_{4} D_{t}(v-1) .
\end{array}
$$

\section{References}

[1] A. Khodayari, S. Pruvost, G. Sebald, D. Guyomar, and S. Mohammadi, "Nonlinear pyroelectric energy harvesting from relaxor single crystals," IEEE Transactions on Ultrasonics, Ferroelectrics, and Frequency Control, vol. 56, no. 4, pp. 693-698, 2009.

[2] D. Guyomar, A. Badel, E. Lefeuvre, and C. Richard, “Toward energy harvesting using active materials and conversion improvement by nonlinear processing," IEEE Transactions on Ultrasonics, Ferroelectrics, and Frequency Control, vol. 52, no. 4, pp. 584-594, 2005.

[3] G. Sebald, E. Lefeuvre, and D. Guyomar, "Pyroelectric energy conversion: optimization principles," IEEE Transactions on Ultrasonics, Ferroelectrics, and Frequency Control, vol. 55, no. 3, pp. 538-551, 2008.

[4] C. Mo, L. J. Radziemski, and W. W. Clark, "Analysis of piezoelectric circular diaphragm energy harvesters for use in a pressure fluctuating system," Smart Materials and Structures, vol. 19, no. 2, Article ID 025016, 2010.

[5] S. Kim, W. W. Clark, and Q. M. Wang, "Piezoelectric energy harvesting using a biomorph circular plate: experimental study," Journal of Intelligent Material Systems and Structures, vol. 16, no. 10, pp. 855-864, 2005.

[6] S. Kim, W. W. Clark, and Q. Wang, "Piezoelectric energy harvesting with a clamped circular plate: analysis," Journal of Intelligent Material Systems and Structures, vol. 16, no. 10, pp. 847-854, 2005.

[7] S. Li and S. Chen, "Analytical analysis of a circular PZT actuator for valveless micropumps," Sensors and Actuators A, vol. 104, no. 2, pp. 151-161, 2003.

[8] C. Mo, R. Wright, W. S. Slaughter, and W. W. Clark, "Behaviour of a unimorph circular piezoelectric actuator," Smart Materials and Structures, vol. 15, no. 4, pp. 1094-1102, 2006.

[9] M. J. Ramsay and W. W. Clark, "Piezoelectric energy harvesting for bio MEMS applications," in Proceedings of the 8th Annual International Symposium on Smart Structures and Materials (SPIE’ 01), vol. 4332, pp. 429-438, 2001.

[10] Y. C. Shu, I. C. Lien, and W. J. Wu, "An improved analysis of the SSHI interface in piezoelectric energy harvesting," Smart Materials and Structures, vol. 16, no. 6, pp. 2253-2264, 2007.

[11] A. Tylikowski, "Control of circular plate vibrations via piezoelectric actuators shunted with a capacitive circuit," Thin-Walled Structures, vol. 39, no. 1, pp. 83-94, 2001. 
[12] S. Roundy, E. S. Leland, J. Baker et al., "Improving power output for vibration-based energy scavengers," IEEE Pervasive Computing, vol. 4, no. 1, pp. 28-36, 2005.

[13] E. Lefeuvre, A. Badel, C. Richard, L. Petit, and D. Guyomar, "A comparison between several vibration-powered piezoelectric generators for standalone systems," Sensors and Actuators A, vol. 126, no. 2, pp. 405-416, 2006.

[14] D. Guyoniar, S. Pruvost, and G. Sebald, "Energy harvesting based on FE-FE transition in ferroelectric single crystals," IEEE Transactions on Ultrasonics, Ferroelectrics, and Frequency Control, vol. 55, no. 2, pp. 279-285, 2008.

[15] G. K. Ottman, H. F. Hofmann, and G. A. Lesieutre, "Optimized piezoelectric energy harvesting circuit using step-down converter in discontinuous conduction mode," IEEE Transactions on Power Electronics, vol. 18, no. 2, pp. 696-703, 2003.

[16] D. Guyomar, G. Sebald, E. Lefeuvre, and A. Khodayari, "Toward heat energy harvesting using pyroelectric material," Journal of Intelligent Material Systems and Structures, vol. 20, no. 3, pp. 265-271, 2009.

[17] H. Sodano, D. Inman, and G. Park, "Comparison of piezoelectric energy harvesting devices for recharging batteries," Journal of Intelligent Material Systems and Structures, vol. 16, no. 10, pp. 799-807, 2005.

[18] T. Starner, "Human-powered wearable computing," IBM Systems Journal, vol. 35, no. 3-4, pp. 618-629, 1996.

[19] T. Starner and J. A. Paradiso, "Human generated power for mobile electronics," in Low Power Electron, C. Piguet, Ed., pp. 1-35, CRC Press, Boca Raton, Fla, USA, 2004.

[20] P. Niu, P. Chapman, R. Riemer, and X. Zhang, "Evaluation of motions and actuation methods for biomechanical energy harvesting," in Proceedings of the IEEE 35th Annual Power Electronics Specialists Conference (PESC '04), pp. 2100-2106, Aachen, Germany, June 2004.

[21] S. Suzuki, T. Katane, H. Saotome, and O. Saito, "Electric power-generating system using magnetic coupling for deeply implanted medical electronic devices," IEEE Transactions on Magnetics, vol. 38, no. 5, pp. 3006-3008, 2002.

[22] S. Suzuki, T. Katane, and O. Saito, "Fundamental study of an electric power transmission system for implanted medical devices using magnetic and ultrasonic energy," Journal of Artificial Organs, vol. 6, no. 2, pp. 145-148, 2003.

[23] W. W. Clark and M. J. Ramsay, "Smart material transducers as power sources for MEMS devices," in Proceedings of the International Symposium on Smart Structures and Microsystems, pp. 19-21, Hong Kong, October 2000.

[24] D. Guyomar, S. Mohammadi, and C. Richard, "Effect of boundary (support) conditions on piezoelectric damping in the case of SSDI vibration control technique," Mechanical Systems and Signal Processing, vol. 23, no. 2, pp. 501-513, 2009.

[25] E. Ventsel and T. Krauthammer, Thin Plates and Shells Theory Analysis and Applications, Marcel Dekker, New York, NY, USA, 2001.

[26] IEEE standard 176, IEEE Standards on Piezoelectricity, Institute of Electrical and Electronics Engineers, New York, NY, USA, 1978. 

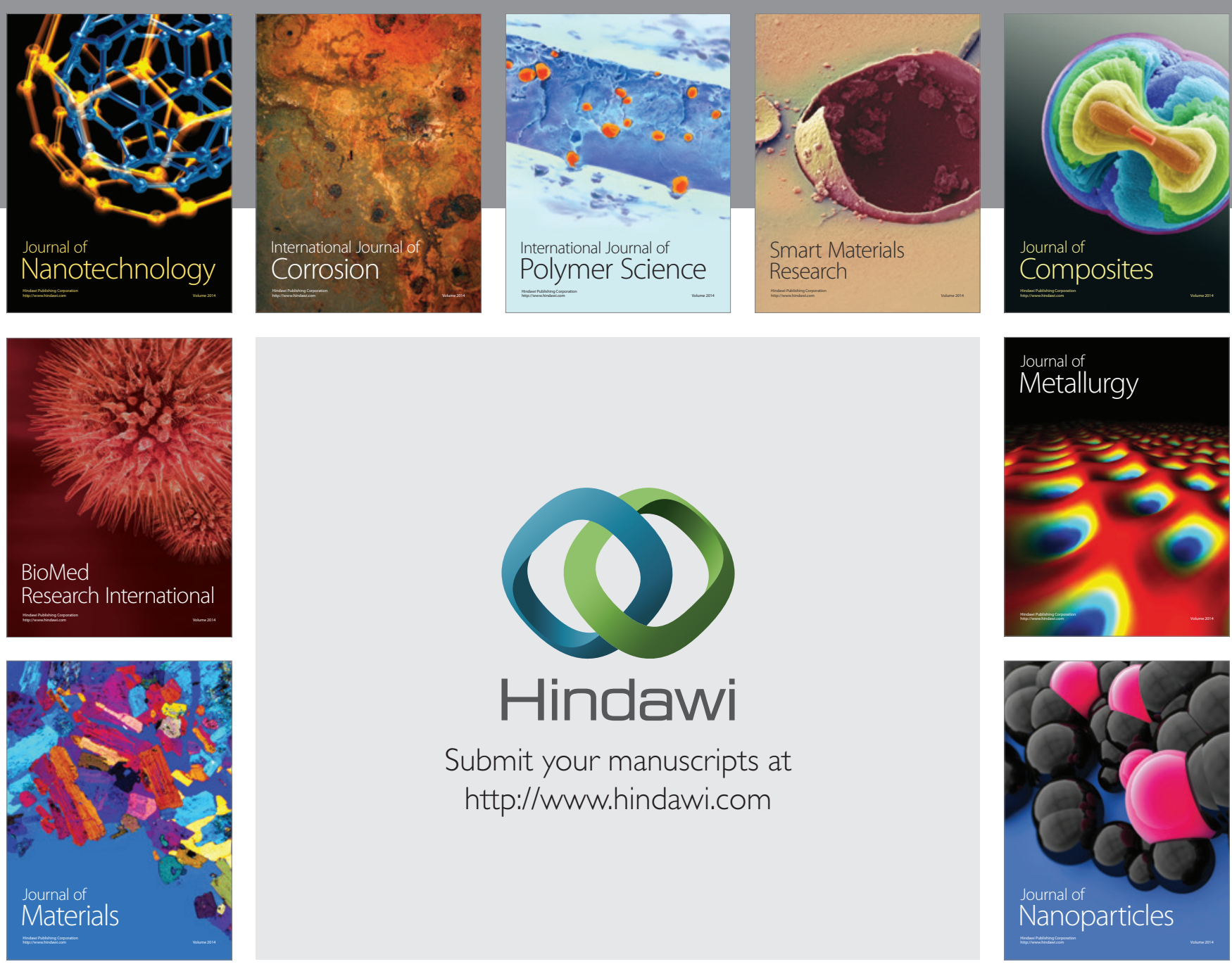

Submit your manuscripts at http://www.hindawi.com
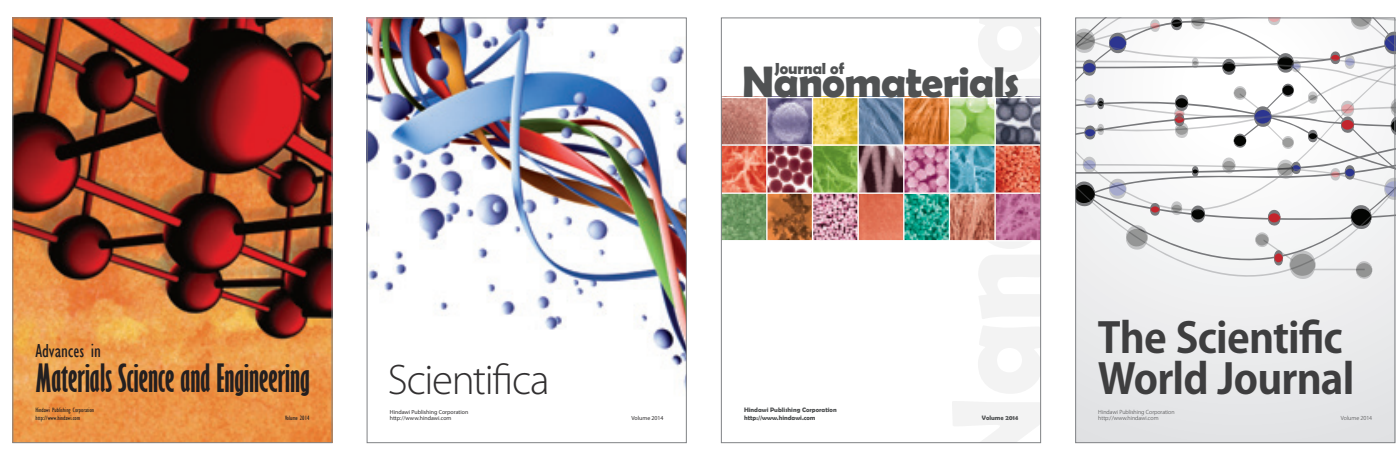

\section{The Scientific World Journal}
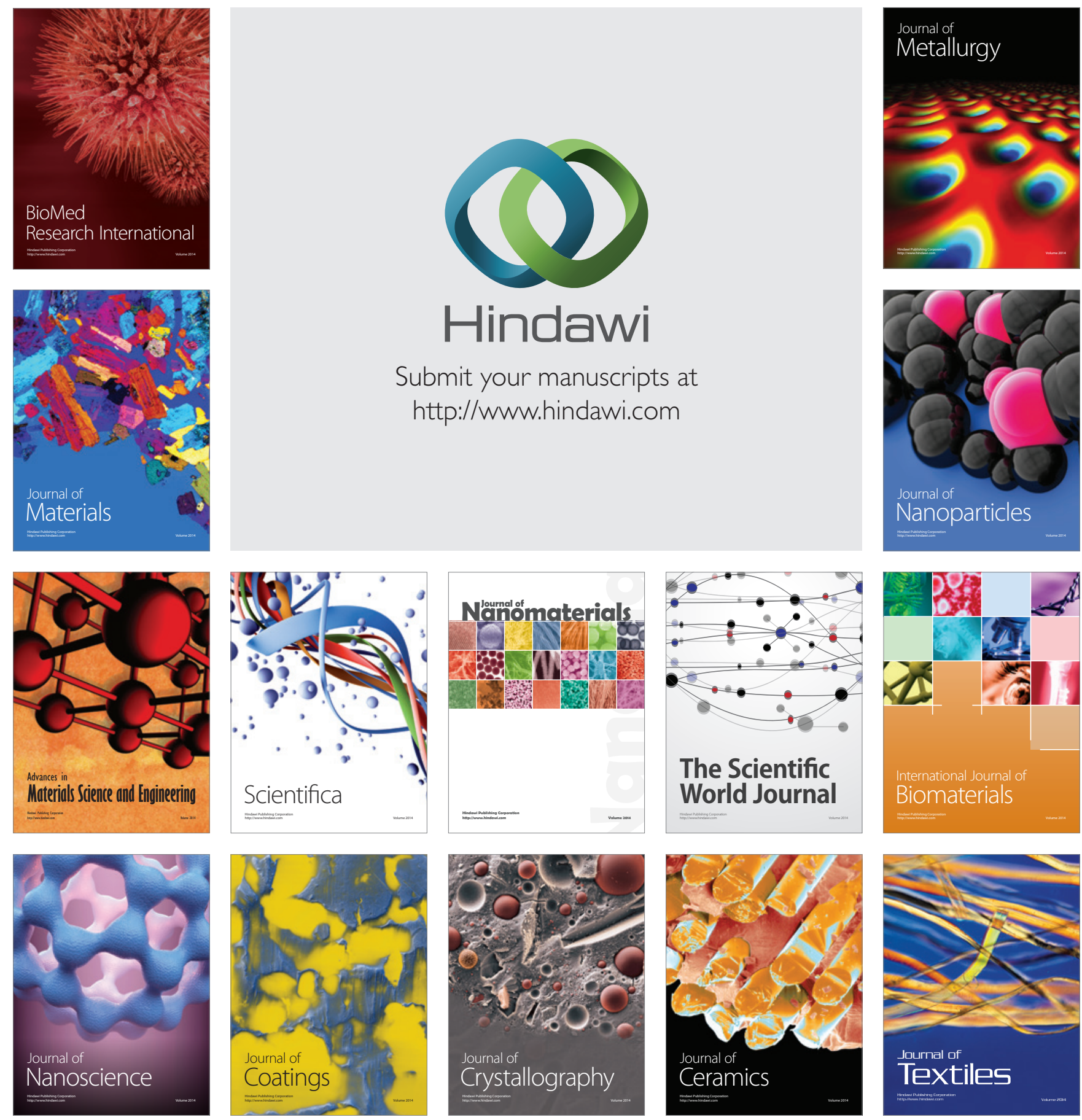